\title{
Rapid intraoperative detection of residual glioma cell in resection cavity walls using a compact fluorescence microscope
}

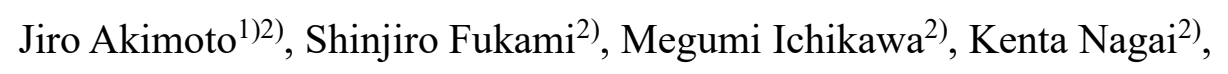
and Michihiro Kohno ${ }^{2)}$

1. Department of Neurosurgery, Kohsei Chuo General Hospital, Tokyo, Japan

2. Department of Neurosurgery, Tokyo Medical University, Tokyo, Japan

Corresponding author: Jiro Akimoto, M.D., Ph.D.

Department of Neurosurgery, Kohsei Chuo General Hospital

Address: 1-11-7 Mita, Meguro-ku, Tokyo 153-8581, Japan

E-mail: jiroaki@gmail.com 


\begin{abstract}
:
Objective: Surgical eradication of malignant glioma cells is theoretically impossible. Therefore, reducing the number of remaining tumor cells around the brain-tumor interface (BTI) is crucial for achieving satisfactory clinical results. The usefulness of fluorescence-guided resection for the treatment of malignant glioma was recently reported, but the detection of infiltrating tumor cells in the BTI using a surgical microscope is not realistic. Therefore, we developed an intraoperative rapid fluorescence cytology system, and evaluated its clinical feasibility for the management of malignant glioma.
\end{abstract}

Materials and methods: Twenty-five selected patients with malignant glioma (newly diagnosed: 17; recurrent: 8) underwent surgical resection under photodiagnosis using photosensitizer Talaporfin sodium and a semiconductor laser. Intraoperatively, a crush smear preparation was made from a tiny amount of tumor tissue, and the fluorescence emitted upon 620/660 nm excitation was evaluated rapidly using a compact fluorescence microscope in the operating theater.

Results: Fluorescence intensities of tumor tissues measured using a surgical microscope correlated with the tumor cell densities of tissues evaluated by measuring the red fluorescence emitted from the cytoplasm of tumor cells using a fluorescence microscope. A "weak fluorescence" indicated a reduction in the tumor cell density, whereas "no fluorescence" did not indicate the complete eradication of the tumor cells, but indicated that few tumor cells were emitting fluorescence.

Conclusion: The rapid intraoperative detection of fluorescence from glioma cells using a compact fluorescence microscope was a useful to evaluate the presence of tumor cells in the resection cavity walls, and provides surgical implications for the more complete resection of malignant gliomas.

Key words: intraoperative photodiagnosis, malignant glioma, fluorescence-guided surgery, intraoperative cytology, fluorescence microscope 


\section{Introduction:}

In the management of the malignant gliomas, the extent of the surgical resection is the most important prognostic factor. [1-5] Therefore, methods to improve the surgical resection rate of these tumors is an important issue for neurosurgeons. Neurosurgeons perform the detailed assessments of the various information such as a patient's clinical profile, the preoperative radiological images, the intraoperative physiological brain functions, the functional brain mapping by awake craniotomy, the optical navigation and intraoperative MRI to resolve this important clinical issue. [1-6]

Recently, the surgical usefulness of the fluorescence-guided resection (FGR) method using the photosensitizer, 5-aminolevulinic acid (5-ALA) has been accepted worldwide, and neurosurgeons confront the malignant gliomas with this novel intraoperative method to improve the extent of resection of these tumors. [7-9] However, with the spread of the FGR method, its problems and limitations have become clear, such as the problem of specificity of 5-ALA accumulation in tumor cells, and the problem of the subjective assessment of the intensity of fluorescence by the surgeon's naked eyes under the surgical microscope. [9-10] Therefore, basic and clinical studies on quantification of the 5-ALA metabolite protoporphyrin IX (PPIX) in the brain and tumor tissues using optical spectroscopy have been performed, and intraoperative augmentation methods of this information using artificial intelligence have been developed to overcome these problems and limitations of FGR. [11-15]

We have been investigating the possibilities of performing the FGR method using another photosensitizer, talaporfin sodium (TPS), for malignant gliomas, and reported the selective accumulation of TPS in glioma cells in both in vitro and in vivo studies, and developed an intraoperative photodiagnosis method using TPS with semiconductor laser systems. [16-20] Furthermore, we reported that additional intraoperative photodynamic therapy (PDT) to the wall of the resection cavity of malignant gliomas using TPS with a semiconductor laser improves the median of progression-free survival and overall survival of patients, and PDT was subsequently approved for use under insurance by the Japanese Government in 2013. [21-23]

Basic research data demonstrated that the intensity of fluorescence emitted from the tumor tissues of a rat xenograft model administrated the TPS was 5 to 8 folds greater than 
that from a tumor model of 5-ALA administration [24], and we reported the detection of strong fluorescence intensities emitted from the tumor tissues in our clinical surgeries of malignant gliomas. [19] However, we encountered problem in the subjective assessment of fluorescence emission intensities of tumors and brain-tumor interfaces (BTI) by the naked eyes under a surgical microscope, similarly to with the evaluation of PPIX. [19,20]

To overcome this problem, we developed an intraoperative rapid fluorescence cytology system for the objective assessment of fluorescence emission from the tumor cells, which take up TPS, in the operative theater. In this article, we introduce the detailed method of this system, and report its clinical feasibility for improvement of the extent of resection of the malignant gliomas, with the demonstration of clinical cases.

\section{Materials and methods:}

\subsection{TPS and semiconductor laser}

The photosensitizer TPS (Laserphyrin ${ }^{\circledR}$, Meiji Seika Pharma Co., Ltd., Tokyo, Japan) is a hydrophilic compound synthesized by coupling aspartic acid and chlorine, and is utilized in PDT for primary malignant brain tumors, in combination with a semiconductor laser (PD Laser BT ${ }^{\circledR}$, Meiji Seika Pharma Co., Ltd., Tokyo, Japan) at a wavelength of 664 $\mathrm{nm}$. TPS is a second-generation photosensitizer that is more quickly excreted from the body than the first-generation photosensitizers, and is characterized by the rapid resolution of the skin photosensitivity reaction.

\subsection{Intraoperative photodiagnosis using a surgical microscope}

TPS was administered as a bolus intra-venously at a dose of $40 \mathrm{mg} / \mathrm{m}^{2}$ in light-shielded conditions, $24 \mathrm{~h}$ prior to surgery. Craniotomy was performed under illumination at $\leq 500$ lux or less. First, the brain surface was observed under halogen light illumination. Then, the halogen light was turned off, and the brain surface was irradiated with a diode laser at $664 \mathrm{~nm}$ at a power density of $10 \mathrm{~mW} / \mathrm{m}^{2}$, with a beam diameter of $40 \mathrm{~mm}$ and an irradiation area of $12.6 \mathrm{~cm}^{2}$, to observe the presence or absence of tumor fluorescence. The wavelength of fluorescence emitted from tumor tissues is $672 \mathrm{~nm}$, and the fluorescence intensity displayed on the monitor was classified into 3 grades: strong fluorescence (S), weak fluorescence (W), and no fluorescence (N). Tumor resection was performed by fluorescence image guidance using an optical navigation system (Brain Lab 
KK, Tokyo, Japan) under physiological monitoring.

\subsection{Compact fluorescence microscope (Fig. 1)}

We used a compact fluorescence microscope BZ-8000 (Biozero ${ }^{\circledR}$, height: $41 \mathrm{~cm}$; width: 31.2cm; depth: $55.7 \mathrm{~cm}$; weight: $28 \mathrm{~kg}$ ) developed by Keyence Co., Ltd. (Osaka, Japan) was used in this study. The microscope was brought into the operating theater and put it in front of the surgeon. (Fig.1 B) This microscope is a handstand type phase-contrast fluorescence microscope containing a dark room box, and has high quality performance with full electric control. The following 3 filter systems were used to observe fluorescence emission from the tumor tissues. PDD-B (excitation: 450/40 nm; absorption:460 nm; dichromic mirror: $435 \mathrm{~nm}$ ) was used to observe autofluorescence from the normal brain, PDD-A (excitation: 550/40 nm; absorption: $610 \mathrm{~nm}$; dichromic mirror: 595nm) for PPIX fluorescence and autofluorescence from 5-ALA metabolites, and Cy5 (excitation: 620/60 nm; absorption: 700/75nm; dichromic mirror: $660 \mathrm{~nm}$ ) for TPS. (Fig. 1 A) The images obtained using each filter system were displayed on the PC monitor using BZ Viewer ${ }^{\circledR}$ (Keyence Co., Ltd., Osaka, Japan), and the surgeons evaluated the presence/absence of fluorescence-emitting tumor cells fluorescence with their own eyes. (Fig. 1 C)

\subsection{Real-time, intraoperative fluorescence cytology}

\subsubsection{Tissue preparation}

Crush smear tissue preparations were made using 2 nonfluorescent glass slides (Matsunami Glass Ind., Ltd., Osaka, Japan) for a 1-mm piece of tumor obtained under photodiagnosis. Immediately, tissues were fixed using aerosol tissue fixative (Cytokeep $\mathrm{II}^{\circledR}$, Alfresa Pharma Co., Ltd., Osaka, Japan) and covered with a nonfluorescent cover glass (Matsunami Glass Ind., Ltd.).

\subsubsection{Tissue assessment by BZ-8000}

The slide glass mounted with fixed tumor tissue was placed in the stage of the dark room of BZ-8000, and images depicted on the monitor of a personal computer (PC, Windows XP) were observed. Movement of the stage, reduction of the scattering light, focusing, zooming (x10 - x400), taking pictures, and changing of the selected filter systems were controlled by the PC. The time required to image observation from tissue 
sampling was less than 2 minutes. After observation of the fluorescence image, hematoxylin and eosin staining of the fixed tissue was performed and histopathological features of the tissue were observed under white light.

\subsubsection{Analysis of fluorescence images}

The obtained fluorescence images on BZ Viewer ${ }^{\circledR}$ were analyzed by the original software, BZ-II analyzer ${ }^{\circledR}$ developed by Keyence Co, Ltd. In this study, we counted the number of cells emitting red fluorescence under the Cy5 filter in x100 images.

\subsection{Phantom experiments}

Experiments were performed to analyze whether the newly developed fluorescence microscopy system enable adequate intraoperative observation of the fluorescence emitted from glioma cells during glioma surgery. TPS at a concentration of $10 \mu \mathrm{g} / \mathrm{mL}$ was mixed with 10\% bovine serum albumin (BSA; Wako Pure Chemical Industries Ltd., Osaka, Japan), and then a cotton fiber was moistened with the solution. This cotton fiber was mounted on the nonfluorescent glass slide and fixed by Cytokeep II $^{\circledR}$. The fixed cotton fiber was covered with a nonfluorescent micro cover glass and then observed the fluorescence emission was observed using Biozero ${ }^{\circledR}$. The other experiment was observation of the autofluorescence of PPIX from iodine egg shells. A small piece (1 x 1 $\mathrm{mm}$ ) of iodine egg shell was mounted onto a glass slide and fixed using Cytokeep II $^{\circledR}$, and then the autofluorescence emitted from the iodine egg shell was observed.

\subsection{Clinical cases (Table 1)}

The study subjects were 25 selected patients who received the protocol-specified surgery after being diagnosed with glioma by preoperative diagnostic imaging performed by a single surgeon (JA) between May 2006 to May 2010. Of the 25 patients with glioma, 17 patients had a newly diagnosed tumor, and 8 patients had a recurrent tumor. The histological malignancy grade was grade II in 1 patient, grade III in 7 patients and grade IV in 17 patients. The institutional review board of Tokyo Medical University approved the study (Study approval number: No.419 and T2020-0278), and all patients gave their informed consent before their participation.

\subsection{Calculation of the extent of resection}

The extent of resection was determined based on MRI images obtained before surgical 
resection and within 3 days after the resection. For gadolinium-enhanced tumors, gadolinium enhanced T1-weighted axial imaging was used. The sum of the products of the perpendicular diameters (SPD) of the contrast-enhanced lesions was calculated. Then, the SPD of the residual lesions on immediate postoperative imaging was determined, and the extent of resection was calculated. For non-gadolinium-enhanced tumors, the SPD of the areas of prolonged T2 on T2-weighted imaging was assessed to calculate the extent of resection.

\subsection{Statistical analysis}

The TPS-positive tumor cell counts in each tumor tissue calculated from the intensity of fluorescence under photodiagnosis were compared by the Student $t$-test, using Mac statistical analysis software ver. 3 (Misumi Co., Ltd., Tokyo, Japan) A $P$-value of less than 0.05 was considered to indicate a statistically significant difference between 2 groups.

\section{Results:}

\subsection{Phantom experiments (Fig. 2)}

After observation of the structure of the cotton fibers under white light, the green autofluorescence from cotton fibers was observed using the PDD-B filter. From the cotton fibers soaked in a mixture of the TPS solutions and 10\% BSA, strong red fluorescence was observed under the PDD-A filter, and the broadband fluorescence could be observed using merged images from the PDD-A and the PDD-B filters. A weak red fluorescence was visible using the Cy 5 filter, which was considered to be the fluorescence emitted from TPS, and not autofluorescence from cotton fiber. (Fig. 2, A-C)

The green autofluorescence of the membrane of the shell of iodine egg shell was visible using the PDD-B filter, and the strong red autofluorescence was observed from the membrane and shell, suspected to be PPIX, using PDD-A filter. Red fluorescence from the PPIX of the shell was also observed using the Cy 5 filter. (Fig.2, D-F)

These findings suggested that the possibility of observing the fluorescence of target molecules within tumor cells using this compact fluorescence microscope together with the appropriate filters.

\subsection{Intraoperative detection of infiltrating tumor cells using fluorescence}




\section{microscopy}

Crush smear tissue samples of the tumor bulk, marginal area of the tumor, and adjacent edematous brain tissue were analyzed using a fluorescence microscope coupled with a Cy 5 filter and $\mathrm{H} \& \mathrm{E}$ staining. The tumor bulk emitted strong fluorescence from the many tumor cells and weak fluorescence from the tumor matrix. In the marginal area, tumor cells emitted strong fluorescence, but the cell count to be less than that of tumor bulk. The tissue of the adjacent edematous brain demonstrated several small cells on $\mathrm{H} \& \mathrm{E}$ staining, however, there was no tumor cells were detected as emitting fluorescence using the Cy 5 filter. (Fig. 3, A-F)

\subsection{Illustrative cases}

Case 11: A 56-year-old woman had a cystic glioblastoma in the right frontal lobe, manifesting as a severe headache. The tumor was resected en bloc using an optical navigation system under continuous motor evoked potential monitoring. The tumor was irradiated using a laser, and the resected tissue of the tumor cyst wall emitted strong red fluorescence upon observation using a surgical microscope, and fluorescence microscopy also demonstrated that many tumor cells in the resected tissue also emitted strong fluorescence. After resection of the tumor bulk, the tissue of surrounding the right frontal horn of the lateral ventricle emitted strong to weak fluorescence, and several tumor cells also emitted fluorescence, and were therefore was additionally resected. On $\mathrm{H}$ \& Estained tissue demonstrated a few atypical cells in the edematous matrix. Postoperative contrast-enhanced MRI demonstrated complete resection of the tumor, and the additional resected area was clearly identified. (Fig. 5, A-H)

Case 10: A 54-year-old man had a glioblastoma in the left temporal lobe, manifesting as a severe headache and a partial seizure. The tumor, which emitted strong fluorescence on surgical microscopy, was resected en bloc using an optical navigation system, and many atypical-shaped tumor cells in the resected tissue also emitted strong fluorescence on the fluorescence microscopy. After resection of the tumor bulk, the tissue of the left hippocampus emitted very weak fluorescence, but several invading several tumor cells emitting strong fluorescence were demonstrated on the fluorescence microscopy, and therefore the hippocampal head was partially resected. Postoperative contrast-enhanced MRI demonstrated complete resection of the tumor, and the additional resected area was clearly identified. (Fig. 6, A-H) 
Case 1: A 30-year-old man had an oligodendroglioma in the left frontal lobe, manifesting as a first episode of generalized tonic and clonic seizures. The tumor was resected en bloc under awake craniotomy, and emitted very weak fluorescence on surgical microscopy, with several tumor cells in the tumor bulk emitting strong fluorescence. However, the merged image of Cy 5 image and PDD-B fluorescence demonstrated a mixture of Cy 5-positive cells and Cy 5-negative cells (mosaic pattern). (Fig. 6, A-D)

Case 18: A 28-year-old man had a recurrent anaplastic oligodendroglioma in the left frontal lobe. The tumor was resected en bloc, which emitted extremely strong fluorescence on surgical microscopy, and many tumor cells in the tumor bulk emitted strong fluorescence. The merged image of Cy 5 and PDD-B fluorescence demonstrated that all tumor cells were Cy 5-positive (diffuse pattern). (Fig. 7, E-H)

\subsection{Association between fluorescence intensity on surgical microscopy and number of cells emitting fluorescence from TPS (Fig. 4)}

We resected the tumor bulk by photodiagnosis using surgical microscopy, and confirmed that the tumor cells emitted the strong fluorescence in the edematous brain matrix with the faint background fluorescence on fluorescence microscopy. There were more than 400 (median: 401; ranage: 75-1,138) strong fluorescence-positive tumor cells in 100 microscopic fields, and large tumor cells showed granular aggregates in the cytoplasm emitting red fluorescence. We were able to confirm the localization of tumor cells by fluorescence emission from their cytoplasm, detected by TPS using the Cy 5 filter in combination with the fluorescence detected using the PDD-B filter. The wall of the resected tumor cavity usually shows weak fluorescence under surgical microscopy, and demonstrate the 50 to 200 (median: 88; range: 34-221) cells emitting the red fluorescence under the faint fluorescent background of the edematous brain matrix. The sizes of the cells emitting red fluorescence in this area were almost uniform. When we confirm the disappearance of fluorescence in the marginal zone of resection on surgical microscopy, we confirmed the presence of fluorescence-positive tumor cells despite their being less than 50 (median: 26; range: 0-71) cells. Therefore, we performed resection of the peritumoral tissue as long as there was no problems on intraoperative physiological monitoring, but only 4 specimens resected from 2 patients in this study showed completely disappearance of fluorescence-emitting tumor cells. We confirmed the statistically significant differences in fluorescence-emitting tumor cell counts among 
tissue resected from the region emitting strong, weak and no fluorescence on surgical microscopy.

\subsection{Improvement in the extent of resection of malignant gliomas using this system}

Evaluation of the extent of resection (EOR) by the postoperative MRI performed within 3 days after surgery demonstrated that the mean EOR of the 25 patients was $94.6 \pm 5.6 \%$, and the median EOR was 98\%. Among the patients with newly diagnosed tumors, the mean EOR was $96.8 \% \pm 3.5 \%$ and the median EOR was $100 \%$, and in the patients with recurrent tumors, the mean EOR was $90 \% \pm 7.3 \%$ and the median EOR was $89.5 \%$. Regarding the patients in whom additional resection was performed according to the intraoperative fluorescence cytology findings despite the disappearance of fluorescence under surgical microscopy, there were 4 cases of newly diagnosed patients and 1 recurrent patient. In these patients, the postoperative MRI demonstrated " supra-total resection " of the tumor.

\section{Discussion:}

In Wilson's scheme of the degree and the distribution of the intracerebral invasion of glioblastoma cells, he suggested that the reduction of these invading tumor cells in the BTI is an important factor for improving the extent of resection of glioblastoma tissue. [25] Conventionally, the extent of resection of the tumor bulk evaluated the gadoliniumenhanced postoperative MRI was the most important prognostic factor. [7-10] The target of FGR using 5-ALA was the tumor bulk demonstrating the gadolinium-enhancement on MRI, and this procedure was clinically approved as improving the progression-free survival of glioblastoma patients, but did nots prolong the overall survival of these patients. [7] Therefore, in glioblastoma surgery, as much as possible of the surrounding tissues infiltrated by tumor cells should be resected, up to the boundary of the functional region of the brain. [8-10,25] Recently, the significance of aiming at the "supra-total" resection, which is resection of tissue containing the invading glioblastoma cells in the area demonstrating a high signal on the preoperative FLAIR imaging beyond the gadolinium-enhanced tumor bulk was reported. [5] However, the subjective evaluation of fluorescence emission by the naked eye, the problems with the sensitivity and specificity of PPIX accumulation within tumor cells, and the autofluorescence of PPIX from normal 
brain tissue were important clinical issue in performing the FGR using 5-ALA, particularly for additional resection of the region demonstrating "vague" fluorescence around the tumor bulk emitting strong fluorescence. [8-15] There have numerous discussions regarding these unresolved problems to date.

Recently, several attempts were performed to resolve these crucial issues of FGR to identify infiltrating the glioblastoma cells in BTI intraoperatively. For instance, the difference in the wavelength of the autofluorescence emitted from the normal brain tissue and brain tumor tissue has been analyzed [26-28], and emission "vague" fluorescence measured using the handheld-type spectrometer and confocal microscope in situ have been analyzed. [29-39] However, the normal brain emits autofluorescence of 3 wavelengths, originating from $\mathrm{NAD}(\mathrm{P}) \mathrm{H}$ (nicotinamide adenine dinucleotide) (ex. 360$380 \mathrm{~nm}$, em. 450-500 nm), FAD (flavin adenine dinucleotide) (ex. 440-450 nm, em. 500$550 \mathrm{~nm}$ ) and porphyrin (mainly PPIX) (ex.490-500 nm, em. 590-630 nm). [40] However, these wavelengths are strongly affected by the absorption by hemoglobin, and therefore, it was not easy to detection of the tumor infiltration using autofluorescence analysis. [2628] In addition, it was also considered that the evaluation using the handheld-type spectrometer and confocal microscopy was affected by the PPIX autofluorescence of the normal brain. [29-31] Furthermore, we have some concern regarding the stability of intraoperative data acquisition and using these handheld-type compact devices. In this regard, our system appears to be simple and consistent for evaluating fluorescence emission of TPS from glioblastoma cells, because it has a wavelength range that is longer than the autofluorescence of the normal brain, which is called the "optical window", indication that it is minimally affected by the absorption of hemoglobin. We were in fact able to distinguish between various types of autofluorescence and the fluorescence emission of TPS from the cotton fiber and iodine egg shell by using the 3-filter system in the phantom experiment. In addition, in the operating theater, we were able to clearly evaluate the autofluorescence of brain tissue and the fluorescence emission of TPS from glioblastoma cells on the PC monitor using the Cy5 filter system. Furthermore, the method of tissue preparation and the procedure of fluorescence microscopy are extremely simple, easy, and stable, and the results can be confirmed within 2 minutes after a biopsy. In addition, all the staff, including the surgeon performing the surgery, can confirm the presence of tumor cells emitting red fluorescence, and can objectively determine the necessity of additional resection of the brain tissue. Furthermore, if a biopsy was 
performed on an area concluded as having no fluorescence by macroscopic photodiagnosis under surgical microscopy, and cells emitting the red fluorescence were still present under fluorescence microscopy [19,20], we performed additional resection if possible according to the patient's neurophysiological evaluation, using PDT in functional brain areas.

In the patients in this study, additional resection was performed on all patients according to the findings on fluorescence microscopy after macroscopic photodiagnosis, up to the limit of the resection evaluated by the intraoperative functional monitoring and navigation. MRI within 3 days after surgery demonstrated the area of additional resection, and we believe we achieved almost supra-total resection in all of our glioblastoma patients.

One patient with low-grade glioma demonstrated interesting findings regarding fluorescence-emitting cells. Almost all malignant gliomas, all tumor cells of the tumor bulk demonstrated TPS uptake (diffuse pattern), but the tumor cells of the low-grade glioma were a mixture of the cells emitting TPS and those not emitting TPS (mosaic pattern). This finding indicates the one of the basic mechanisms causing the weak or no fluorescence image on macroscopic photodiagnosis of low- grade glioma. [19,20,39] TPS, which is a water-soluble photosensitizer, promptly conjugates to albumin after its intravenous administration and funnels the blood vessel. [41] TPS is then distributed in the interstitial tissue after direct leakage from the disrupted blood-brain barrier (BBB) or leakage by bulk flow into the interstitial fluid, called the enhanced permeability and retention effect, and is taken up into tumor cells by the transporter SLC46A1, which is a heme carrier protein that accumulates in the lysosomes of tumor cell. [42] In this fluorescence microscopic study, strong red fluorescence was detected strongly from the cytoplasm of the tumor cells, and faint fluorescence was also detected from the interstitial tissue of the tumor. The faint fluorescence might be emitted from the remnant TPS in the edematous interstitial tissue of the tumor which was not taken up by the tumor cells. In low-grade gliomas, faint fluorescence was also emitted from the interstitial tissue, but the tumor cells demonstrated a mosaic uptake pattern of TPS, which suggests the possibility of a heterogeneous uptake mechanism in tumor cells. Low-grade gliomas have minimal disruption of the BBB, fewer tumor cells, and lower proliferation potency than highgrade gliomas, and are hence expected to demonstrate less fluorescence from the photosensitizer. $[19,20,42]$ Although our findings suggest that the heterogeneous uptake 
mechanism of TPS plays an important role in the lower fluorescence emission from lowgrade glioma tissue, in the future, further research should be performed to clarify the uptake mechanism of TPS in these tumor cells.

Regarding limitations of this study, evaluations were limited to a small number of patients with glioma who underwent surgery performed by a single surgeon (JA) in a single institution. In the future, evaluations should be performed for all types of primary malignant brain tumors, including malignant meningiomas and malignant skull-base tumors. Furthermore, data of surgeries performed by other surgeons in other institutions should be included from the viewpoint of objectivity, and to promote the spread of this brief, reliable, and low-cost method for intraoperative brain tumor cell detection that we developed.

\section{Conclusion:}

This intraoperative rapid fluorescence cytology system is expected to become useful in many areas of the cancer surgery in the future. As it is known that TPS is taken up and retained in the most cancer cells, this method will offer the crucial additional information for surgeons, such as the confirmation of the accuracy of the biopsy sample, the limit of surgical resection, and the presence of lymph node metastases within an extremely short time. Furthermore, all medical staffs can objectively understand the importace of the surgery by sharing such information. The Japanese government approves the administration of TPS under medical insurance for surgeries for primary lung cancer and the recurrent esophageal cancer after chemoradiotherapy, and primary malignant brain tumor. We believe that our brain tumor cell detection system will useful for all surgeons who treat these types of cancers.

Author Contributions: J.A., writing-original draft preparation; S.Y., M.I., K.N. and M.K., writing-review and editing. All authors have read and agreed to the publish version of the manuscript.

Funding: This research received no external funding.

Institutional Review Board Statement: This research was approved by the Ethics 
Review Board of Tokyo Medical University Hospital (study approval no.: No.419 and T2020-0278).

Informed Consent Statement: Each patient signed an informed consent form that was approved by the Institutional Committee on Human Rights in Research of Tokyo Medical University, for the publication of their data.

Data Availability Statement: The study did not report any data.

Conflicts of Interest: The authors declare that they have no conflicts of interest.

Acknowledgements: The authors are indebted to the medical editors from the Department of International Medical Communications of Tokyo Medical University for editing and reviewing the English manuscript.

\section{Reference:}

1. Lacroix M, Abi-Said D, Fourney DR, et al. A multivariate analysis of 416 patients with glioblastoma multiforme: Prognosis, extent of resection, and survival. J. Neurosurg. 2001, 95, 190-198.

2. Sanai N, Polley MY, McDermott MW, et al. An extent of resection threshold for newly diagnoses glioblastomas. J Neurosurg 2011, 115, 3-8.

3. Chen I, Mao Y. Gross total resection plays a leading role in survival of patients with glioblastoma multiforme. World Neurosurg 2014, 82, 105-107.

4. Grabowski MM, Recinos PF, Nowacki S, et al. Residual tumor volume versus extent of resection: predictors of survival after surgery of glioblastoma. J Neurosurg 2014, 121, 1115-1123.

5. Li YM, Suki D, Hess K, et al. The influence of maximum safe resection of glioblastoma on survival in 1229 patients: can we do better than gross-total resection? J Neurosurg 2016, 124, 977-988.

6. Yordanova YN, Moritz SG, Duffau H. Awake surgery for WHO II gliomas within "noneloquent" areas in the left dominant hemisphere: toward a “supratotal" resections. J Neurosurg 2011, 115, 232-239. 
7. Stummer W, Pichlmeier U, Meinel T, et al. Fluorescence-guided surgery with 5-aminolevulinic acid for resection of malignant glioma: a randomized controlled multicenter phase III trial. Lancet Oncol 2006, 7, 392-401.

8. Liu JTC, Meza D, Sanai N. Trends in fluorescence image guided surgery for gliomas. Neurosurgery 2014, 75: 61-71.

9. Sun R, Cuthbert H, Watts C. Fluorescence-guided surgery in the surgical treatment of gliomas: Past, Present and Future. Cancers 2021, 13, 3508. https://doi.org/10.3390/ cancers 13143508.

10. Stummer W, Tonn JC, Goetz C, et al. 5-Aminolevulinic acid-derived tumor fluorescence: the diagnostic accuracy of visible fluorescence qualities as corroborated by spectrum and histology and postoperative imaging. Neurosurgery 2014, 74: 310-319.

11. Valdes PA, Leblond F, Kim A, et al. Quantitative fluorescence in intracranial tumor: implications for ALA-induced PpIX as an intraoperative biomarker. J Neurosurg 2011, 115, 11-17.

12. Valdes PA, Leblond F, Jacobs VL, et al. Quantitative, spectrally-resolved intraoperative fluorescence imaging. Sci Rep 2012, 2, 798, doi:10.1038/srep00798.

13. Widhalm G, Wolfsberger S, Minchev G, et al. 5-Aminolevulinic acid is a promising marker for detection of anaplastic foci in diffusely infiltrating gliomas with non-significant contrast enhancement. Cancer 2010, 116, 1545-52.

14. Roberts DW, Valdes PA, Harris BT, et al. Coregistered fluorescence-enhanced tumor resection protoporphyrin IX fluorescence, magnetic resonance imaging enhancement, and neuropathological parameters. J Neurosurg 2011, 114, 595603.

15. Widhalm G, Kiesel B, Woehrer A, et al. 5-aminolevulinic acid induced fluorescence is a powerful intraoperative marker for precise histopathological grading of gliomas with non-significant contrast-enhancement. PLoS ONE 2013, 8, e76988.

16. Tsutsumi M, Miki Y, Akimoto J, et al. Photodynamic therapy using talaporfin sodium induces dose-dependent apoptotic cell death in human glioma cell lines. Photodiagnosis Photodyn Ther 2013, 10(2), 103-110

17. Matsumura H, Akimoto J, Haraoka J, et al. Uptake and retention of the 
mono-L-asparthyl chlorine e6 in experimental glioma. Lasers Med Sci 2008, $23,235-245$

18. Namatame H, Akimoto J, Matsumura H, et al. Photodynamic therapy of C6-implanted glioma cells in the rat brain employing second-generation photosensitizer talaporfin sodium. Photodiagnosis Photodyn Ther 2008, 5(3), 198-209

19. Akimoto J, Fukami S, Ichikawa M, et al. Intraoperative photodiagnosis for malignant glioma using photosensitizer talaporfin sodium. Front Surg 2019,6, 12.

20. Shimizu K, Nitta M, Komori T, et al. Intraoperative photodynamic diagnosis using talaporfin sodium simultaneously applied for photodynamic therapy against malignant glioma: A prospective clinical study. Front Neurol 2018, Jan 30; 9:24. doi: 10.3389/fneur. 2018.00024. eCollection 2018.

21. Akimoto J, Haraoka J, Aizawa K. Preliminary clinical report of safety and efficacy of photodynamic therapy using Talaporfin sodium for malignant gliomas. Photodiagnosis Photody Ther 2008, 5, 198-209.

22. Muragaki Y, Akimoto J, Maruyama T, et al. Phase II clinical study on intraoperative photodynamic therapy with talaporfin sodium and semiconductor laser in patients with malignant brain tumors. J Neurosurg 2013, 119, 845-852.

23. Nitta M, Muragaki Y, Maruyama T, et al. Role of Photodynamic therapy using talaporfin sodium and a semiconductor laser in patients with newly diagnosed glioblastoma. J Neurosurg 2018, Dec 7: 1-8. Doi: 10.1016/j.wneu. 12.095.

24. Tsurubuchi T, Zoboronok A, Yamamoto T, et al. The optimization of fluorescence imaging of brain tumor tissue differentiated from brain edema- In vivo kinetic study of 5-aminolevuinic acid and talaporfin sodium.

Photodiagnosis Photodyn Ther 2009, 6, 19-27.

25. Wilson CB. Glioblastoma; the past, the present, and the future. Clin Neurosurg 1992, 38, 32-48.

26. Bottiroli G, Croce AC, Locatelli D, et al. Brain tissue autofluorescence: an aid for intraoperative delineation of tumor resection margins. Cancer Detection Prevention 1998, 22(4), 330-339.

27. Butte PV, Fang Q, Javier A., Intraoperative delineation of primary brain tumors using time-resolved fluorescence. J Biomed Opt 2010, 15(2): 027008. 
28. Croce AC, Fiorani S, Locatelli D, et al. Diagnostic potential of autofluorecence for an assisted intraoperative delineation of glioblastoma resection margins. Photochem Photobiol 2013, 77(3), 309-318.

29. Sanker T, Delaney PM, Ryan RW, et al. Miniaturized handheld confocal microscopy for neurosurgery; Results in an experimental glioblastoma model. Neurosurgery 2010, 66, 410-418.

30. Mitra S, Foster TH. In vivo confocal fluorescence imaging of the intratumor distribution of the photosensitizer mono-L-aspartylchlorine-e6.

Neoplasia 2008, 10, 429-438.

31. Sanai N, Snyder LA, Honea NJ, et al. Intraoperative confocal microscopy in the visualization of 5-aminolevulinic acid fluorescence in low grade gliomas. J Neurosurg 2011, 115, 740-748.

32. Wei L, Fujita Y, Sanai N, et al. Toward quantitative neurosurgical guidance with high-resolution microscopy of 5-aminolevulinic acid-induced protoporphyrin IX. Front Oncol 2019, 9, 592. doi:10.3389/fonc.00592.

33. Wei L, Chen Y, Yin C, Borwege S, et al. Optical-sectioning microscopy of protoporphyrin IX fluorescence in human gliomas. standardization and quantitative comparison with histology. J Biomed Opt 2017, 22, 46005. doi: 10.1117/1. JBO.22.4.046005.

34. Kantelhardt SR, Leppert J, Krajewski J, et al. Imaging of brain and brain tumor specimes by time-resolved multiphoton excitation microscopy ex vivo.

Neuro-Oncology 2007, 9, 103-112.

35. Roberts DW, Olson JD, Evans LT, et al. Red-light excitation of protoporphyrin IX fluorescence for subsurface tumor detection. J Neurosurg 2017, Aug4, 1-8, doi:10.317/2017.1. 162061.

36. Asai K, Sumiyama Y, Watanabe M, Aizawa K. Tumor viability using real-time spectral images. Surg Today 2006, 36, 1075-1084.

37. Valdes PA, Jacobs V, Harris BT, et al. Quantitative fluorescence using 5-aminolevuinic acid-induced protoporphyrin IX biomarkers as a surgical adjunct in low-grade glioma surgery. J Neurosurg 2015, 123, 771-780.

38. Lau D, Hervey-Jumper SD, Chang S, et al. A prospective phase II clinical trial of 5-aminolevulinic acid to assess the correlation of intraoperative fluorescence intensity and degree of histologic cellularity during resection of high-grade 
gliomas. J Neurosurg 2016, 124, 1300-1309.

39. Toms SA, Lin WC, Weil RJ, et al. Intraoperative optical spectroscopy identifies infiltrating glioma margins with high sensitivity. Neurosurgery 2005 , 57 (ONS Suppl 3): ONS-382-ONS-391.

40. Chung YG, Schwartz Jam Gardner CM, et al. Diagnostic potential of laser-i nduced autofluorescence emission in brain tissue. J Korean Med Sci 1997,12, 135-142.

41. Kessel D. Pharmacokinetics of N-asparthyl chlorine-e6 in cancer patients. J Photochem Photobiol B 1997, 39, 81-83.

42. Takada T, Tamura M, Yamamoto T, et al. Selective accumulation of hematoporphyrin derivative in glioma through proton coupled folate transportor SLCA46A1. J Clin Biochem Nutr 2014, 54, 26-30. 


\section{Figure legends}

\section{Figure 1. Absorption spectrum of TPS and filter systems}
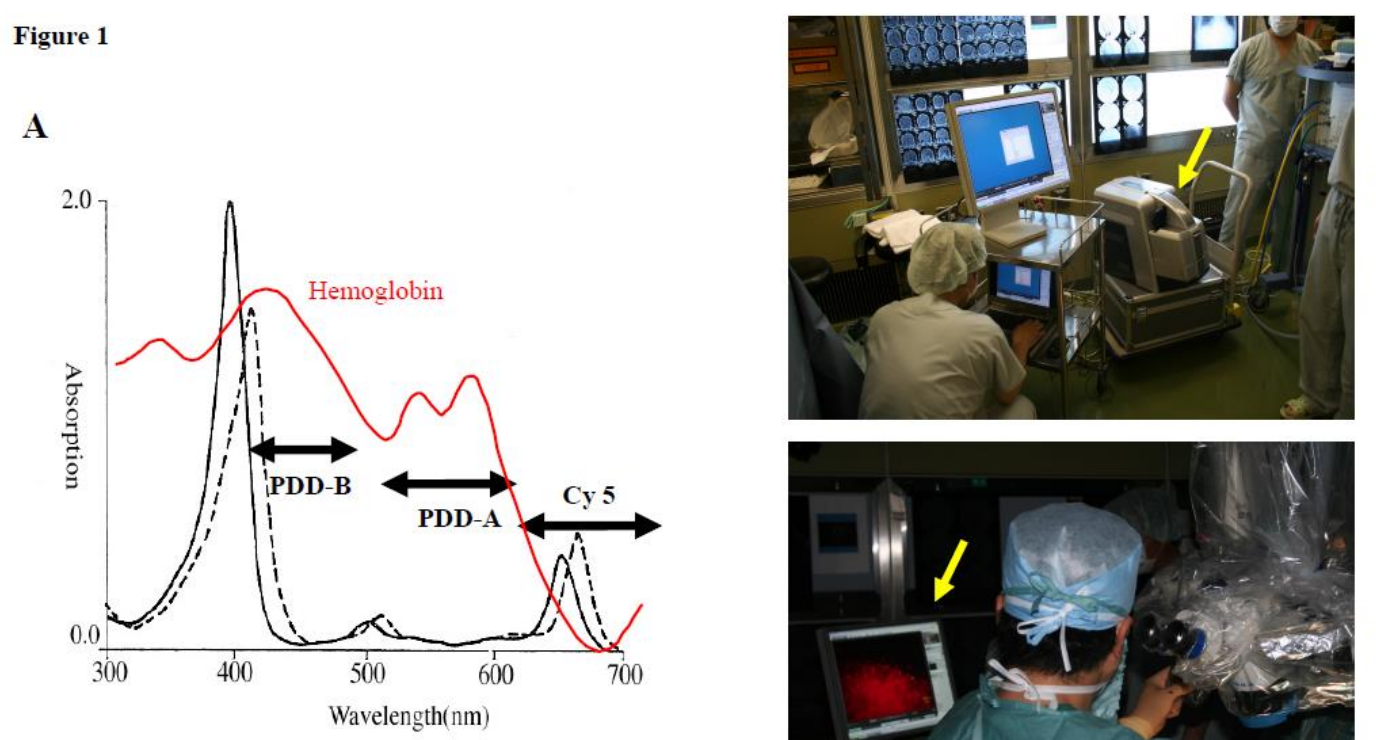

B

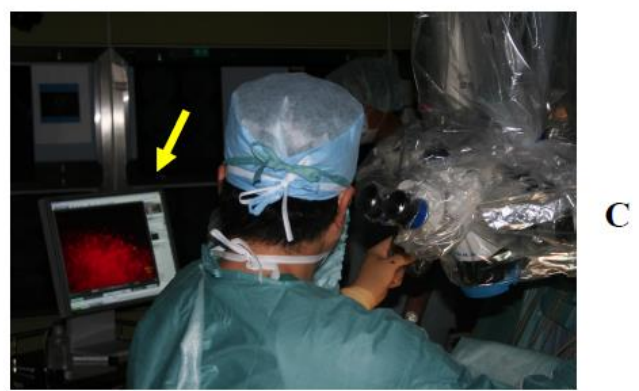

A: Absorption spectrum of TPS and hemoglobin. (solid line: TPS and phosphate buffer solution; dotted line: TPS conjugated with albumin; red line: hemoglobin). Three filter systems were used to observe the fluorescence emitted from the obtained tissues. (PDD-B: excitation 450/40 nm, absorption $460 \mathrm{~nm}$; PDD-A: excitation 550/40 nm, absorption $610 \mathrm{~nm}$;Cy 5: excitation 620/60 nm, absorption $700 / 75 \mathrm{~nm})$.

B: A compact fluorescence microscope (yellow arrow) placed in the operating theater.

C: $\quad$ Fluorescence images obtained under each filter system were displayed on the PC monitor (yellow arrow), and the surgeon was able to detect the presence of the tumor cells emitting fluorescence. 


\section{Figure 2. Phantom experiments}

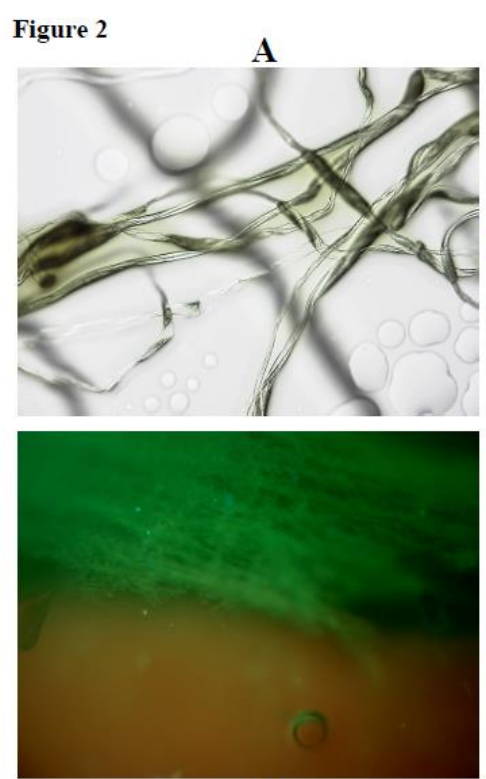

D
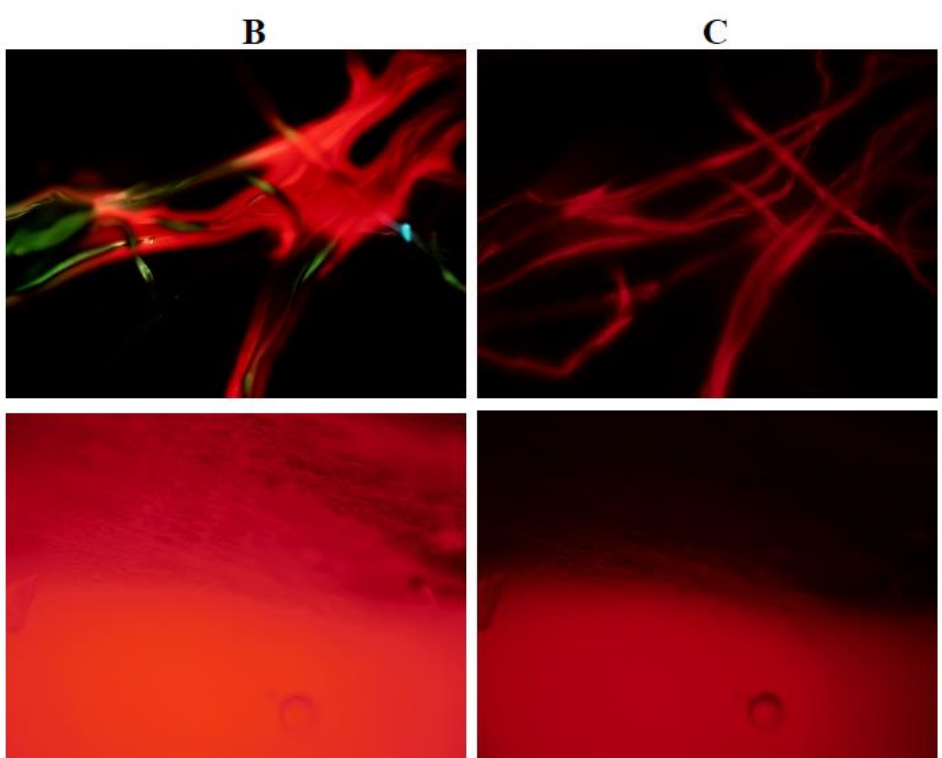

$\mathbf{E}$

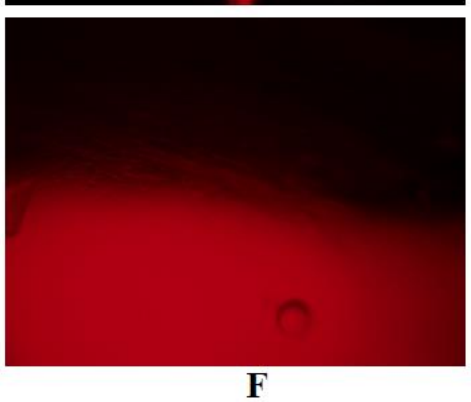

A-C: Cotton fibers were moistened with TPS and bovine serum.

D-F: Autofluorescence of PP IX from an iodine egg shell. A: image under white light; B: merged image under the PDD-B and PDD-A filters; $C$ and F: under the Cy 5 filter; D: under the PDD-B filter; E: under the PDD-A filter. 
Figure 3. Intraoperative detection of fluorescence emittied from tumor tissues and adjacent brain tissue

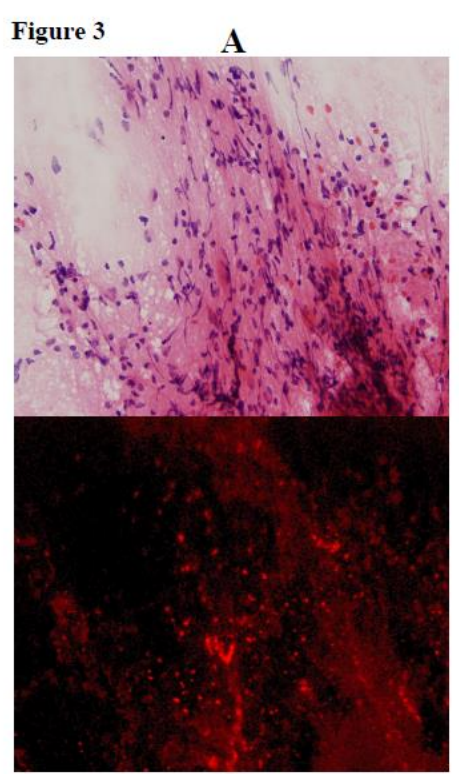

B

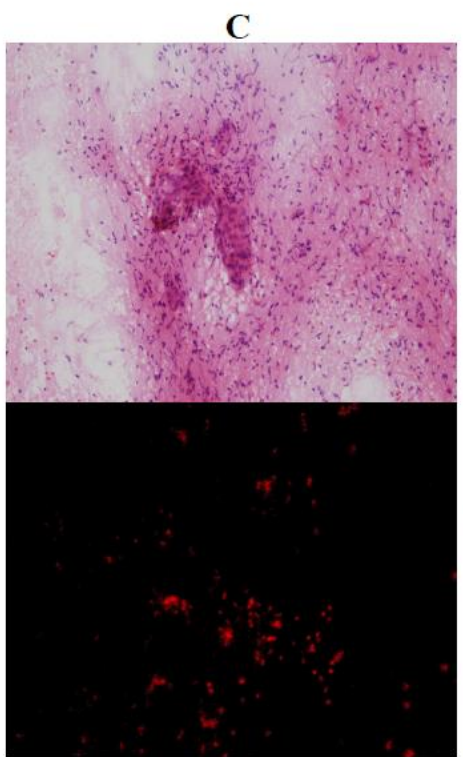

D

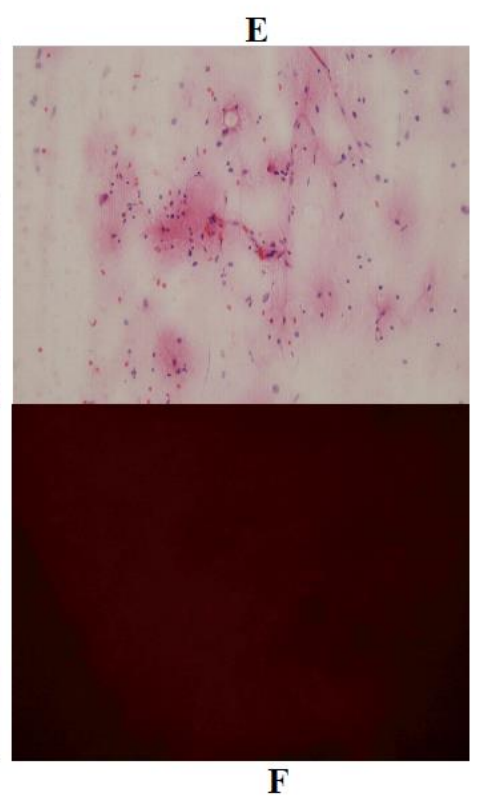

F

A-C. Hematoxylin and eosin staining of crush smear tissue samples of the tumor bulk (A), marginal area of the tumor (B), and adjacent edematous brain tissue (C).

D-F. Fluorescence images under the Cy 5 filter system. A total of 208 cells emitting strong fluorescence were detected in the tissue of tumor bulk (D), 114 cells were detected in the tissue of marginal area (E) and a few cells were detected in the adjacent brain tissue $(\mathrm{F})$. 
Figure 4. Association between fluorescence intensity on photodiagnosis using surgical microscope, and cell count determined by fluorescence emission on fluorescence microscopy

Figure 4

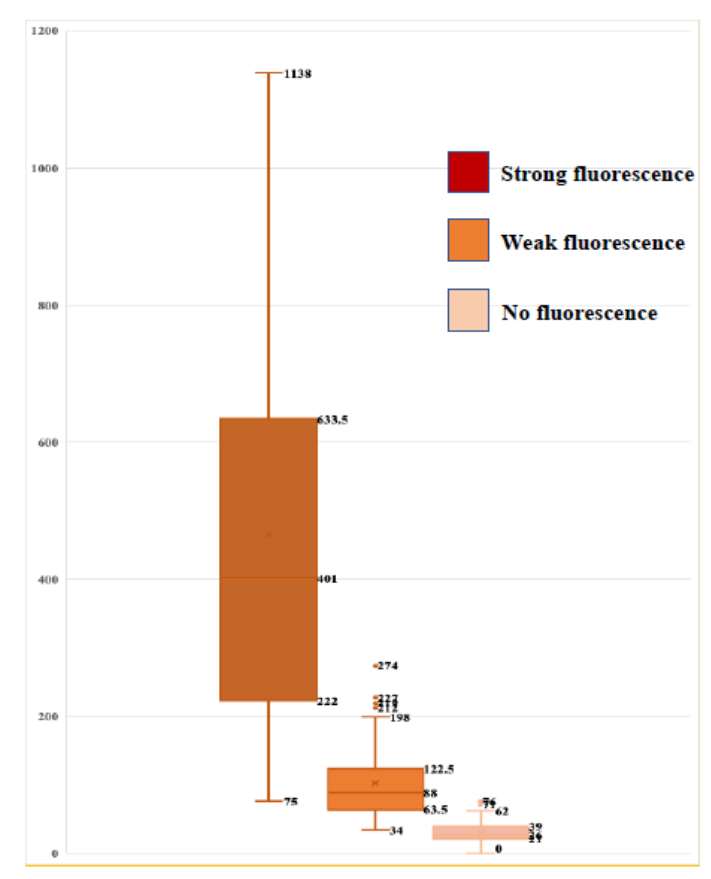

There was a statistically significant difference between the strong fluorescence and the weak fluorescence group $(p<0.001)$, and the weak fluorescence and no fluorescence group $(p<0.05)$. (Student $t$-test) 


\section{Figure 5. Illustrative case 11}

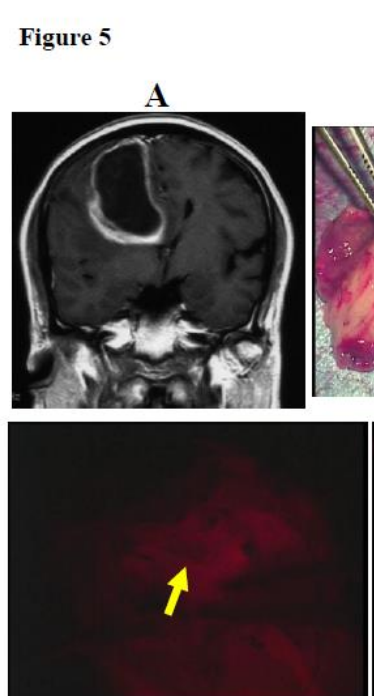

E
B

C
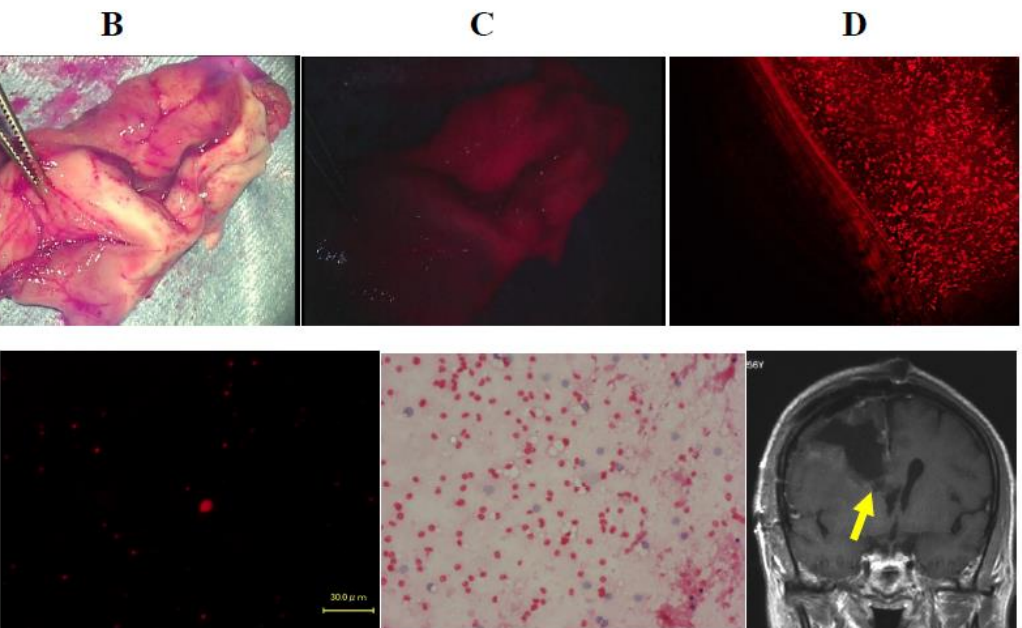

F

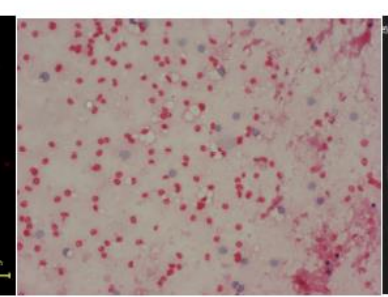

G

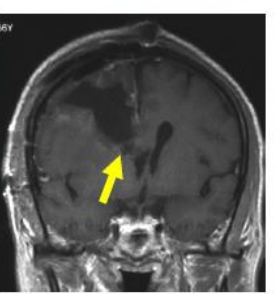

H

A. Gadolinium-enhanced T1-weighted coronal image demonstrating a strongly enhanced cystic glioblastoma lesion in the right frontal lobe.

B. Brain tissue containing the tumor tissue was resected completely using an optical navigation system under continuous motor evoked potential monitoring.

C. Strong fluorescence from the resected tumor tissue was detected under a surgical microscope during photodiagnosis.

D. A total of 761 cells emitting fluorescence were detected in the tumor issue under the fluorescence microscopy using the Cy 5 filter.

E. Weak fluorescence (yellow arrow) was detected in the marginal brain tissue under surgical microscopy.

F. A total of 61 cells emitting fluorescence were detected in the marginal brain tissue under fluorescence microscopy using the Cy 5 filter.

G. Hematoxylin and eosin staining of a crush smear sample of the same tissue same as in $\mathrm{F}$.

H. Gadolinium-enhanced T1-weighted coronal image after surgery demonstrating complete resection of the tumor bulk and the additional resected area (yellow arrow) of the marginal brain tissue. 


\section{Figure 6. Illustrative case 10}

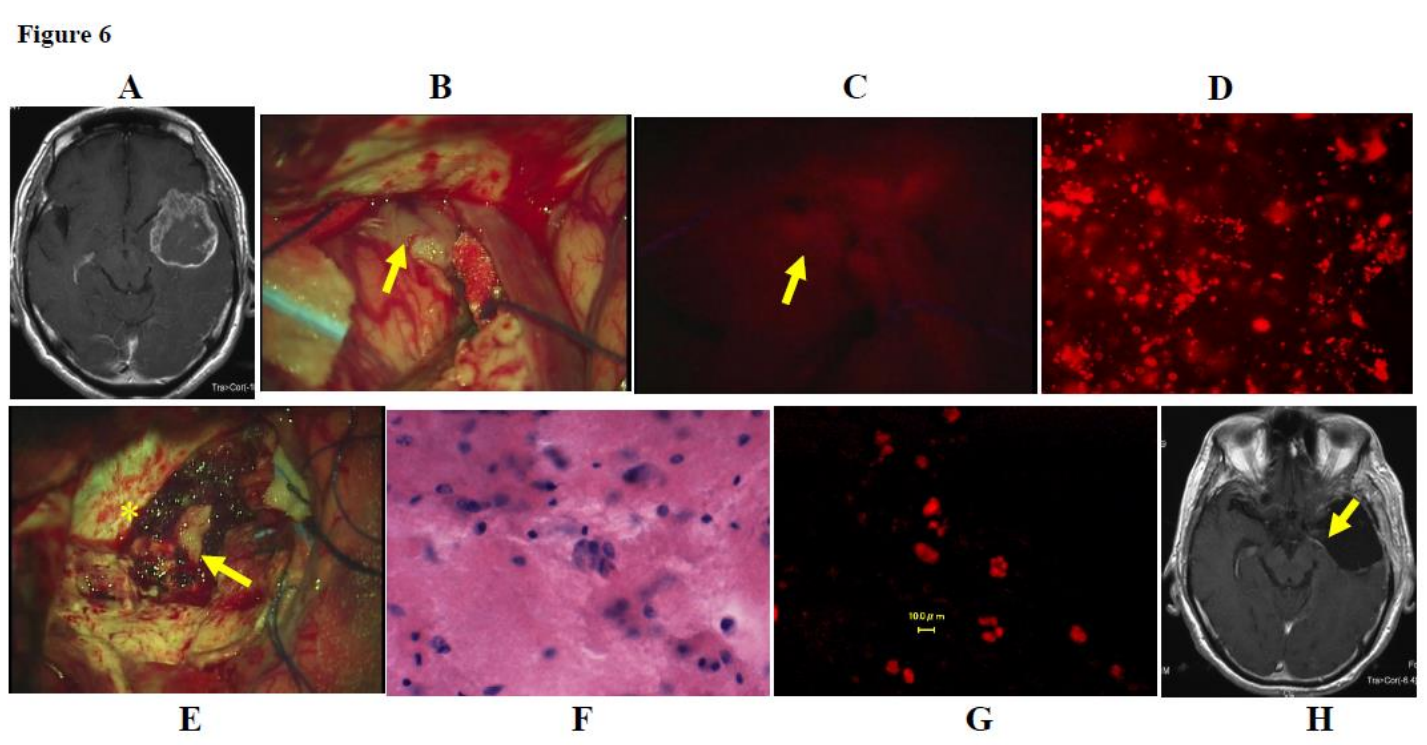

A. Gadolinium-enhanced T1-weighted axial MR image demonstrating a heterogeneously enhanced glioblastoma lesion in the left temporal lobe.

B. The tumor tissue showed exophytic growth in the left temporal lobe (yellow arrow) on the surgical microscopy.

C. Strong fluorescence was detected on surgical microscopy (yellow arrow) for photodiagnosis.

D. A total of 412 polymorphic tumor cells emitting fluorescence were detected in the tumor bulk tissue under fluorescence microscopy using the Cy 5 filter.

E. After resection of the tumor bulk, the hippocampal head (yellow arrow) showed a normal appearance under surgical microscopy. asterisk: tentorial notch

F. Hematoxylin and eosin staining of crush smear tissue of the hippocampal head demonstrated infiltrating multinucleated tumor cells under the white light of the fluorescence microscopy.

G. Infiltrating tumor cells in the tissue in $(\mathrm{F})$ demonstrated strong fluorescence on the fluorescence microscopy using the Cy 5 filter.

H. Postoperative gadolinium-enhanced T1-weighted axial MR image demonstrated complete resection of the tumor bulk and additional resection of the hippocampal head. (yellow arrow). 


\section{Figure 7. Illustrative case 1 (A-D) and case 18 (E-H)}

Fig. 7
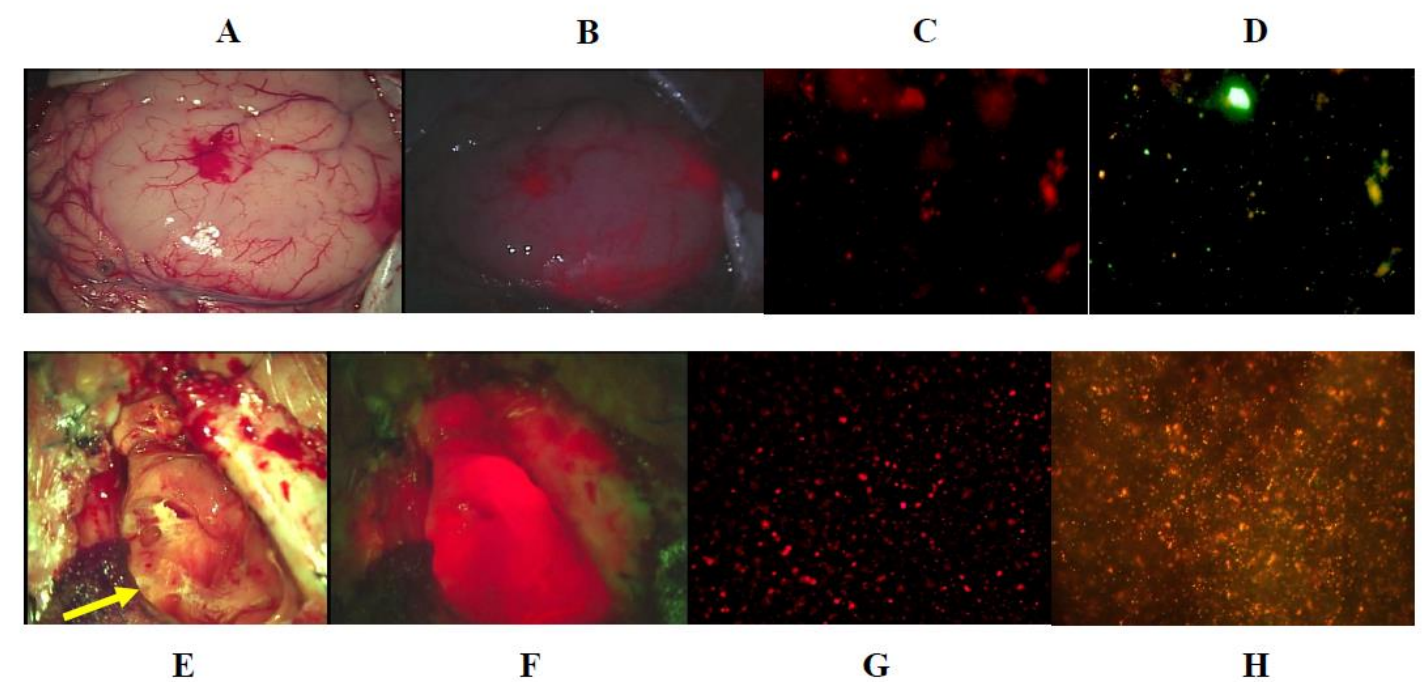

A. The tumor tissue in the cortical gyrus of the left frontal lobe showed hypertrophic features.

B. Weak fluorescence was detected on photodiagnosis using the surgical microscopy.

C. A total of 97 cells emitting fluorescence were detected in the tumor bulk tissue under fluorescence microscopy using the Cy 5 filter.

D. Merged images taken using the Cy 5 filter and the image of using PDD-B filter demonstrated a mosaic pattern of fluorescence-emitting and non-emitting cells.

E. The recurrent tumor tissue was a nodular cortical mass. (yellow arrow).

F. Strong fluorescence was observed on photodiagnosis using surgical microscopy.

G. A total of 573 cells emitting fluorescence were detected in the tumor bulk under fluorescence microscopy using the Cy 5 filter.

H. Merged image of images taken using the Cy 5 filter and PDD-B filter demonstrated a diffuse pattern with almost all tumor cells emitting fluorescence.

\section{Supplementary Table 1.}

\section{Demographic data and TPS fluorescence characteristics in 25 patients with glioma}

N/A: not analyzed, *: macroscopic means intraoperative findings of photo-diagnosis under surgical microscope 